\title{
Depression Und Psychose in der Menopause - KanN eine
} HORMONTHERAPIE HILFREICH SEIN?

\section{Korrespondenzadresse:}

Prof. Dr. med. Anita Riecher-Rössler

Chefärztin

Universitäre Psychiatrische Kliniken Basel

Psychiatrische Universitätspoliklinik /

Allgemeine Psychiatrie ambulant

c/o Universitätsspital Basel

Petersgraben 4

$\mathrm{CH}-4031$ Basel

Tel.: +41612655114

Fax: +41612654599

Email: Anita.Riecher@upkbs.ch

Dieser Beitrag erschien in einer Vorform 2009 in der Zeitschrift Gynäkologie (RiecherRössler A: Psychische Erkrankungen in der Menopause - Depressive Störungen und Psychosen. Gynäkologie 2009;1:15-19) und wird hier mit freundlicher Nachdruckgenehmigung des Rosenfluh-Verlags publiziert. 


\section{Zusammenfassung}

Die Menopause kann bei Frauen das psychische Befinden nachhaltig beeinflussen. Psychische Erkrankungen wie depressive Störungen oder Psychosen treten in dieser Zeit gehäuft auf oder verschlechtern sich im Verlauf. Dies hat wahrscheinlich nicht nur mit den vielen psychosozialen und physiologischen Veränderungen von Frauen in diesem Alter zu tun, sondern ganz speziell auch mit dem Verlust der körpereigenen Östrogenproduktion. Östrogene und hier speziell Estradiol-17- $\beta$ haben wichtige neuro- und psychoprotektive Eigenschaften. In der Therapie dieser Erkrankungen ist daher neben Psychopharmaka, Psychotherapie und sozialen Massnahmen in bestimmten Fällen auch der Ersatz des psychoaktiven Estradiol-17$\beta$ zu erwägen. Dabei sind bestimmte Risiken zu beachten und Indikation bzw. Kontraindikation sorgfältig in Zusammenarbeit mit einem Gynäkologen/einer Gynäkologin abzuklären.

Schlüsselwörter: Menopause, Depression, Psychose, Östrogene, Estradiol 


\section{Abstract}

Depression and psychosis in the menopause - can hormon replacement therapy be helpful?

Menopause can severely influence mental wellbeing of women. Mental disorders such as depression or psychoses show an excess incidence in this time or deterioration of course. This is probably not only due to the manifold psychosocial and physiological changes women have to deal with in this age group, but also specifically with the physiological loss of estrogen production. Estrogens, and specifically estradiol-17- $\beta$ show important neuro- and psychoprotective properties. Regarding therapy of these disorders, besides psychopharmacotherapy, psychotherapy and social measures, in some cases also substitution of estradiol-17$\beta$ has to be taken into account. In this case, certain risks have to be considered and indications as well as contraindications have to be carefully assessed in cooperation with a gynecologist.

Keywords: menopause, depression, psychosis, estrogens, estradiol 


\section{Hintergrund}

Die Menopause ist ein physiologisches Ereignis. Sie geht mit hormonellen und biologischen sowie häufig auch zahlreichen psychosozialen Veränderungen einher. Diese Veränderungen können das psychische Befinden von Frauen nachhaltig beeinträchtigen mit erhöhter Stimmungslabilität, Reizbarkeit, Nervosität und Schlafstörungen, ja psychische Störungen bei vulnerablen Frauen auslösen oder verstärken.

Im Folgenden sollen vor allem depressive Störungen und Psychosen betrachtet werden. Dabei wird auf den Einfluss der Östrogene fokussiert werden und auf die therapeutischen Möglichkeiten, die sich durch Östrogenbehandlung ergeben. Wichtig ist dabei, Menopause von Perimenopause und Postmenopause zu unterscheiden (Tab. 1).

\section{- Tabelle 1 etwa hier einfügen}

\section{Östrogene und Hirnfunktion}

Heute wissen wir, dass Östrogene zahlreiche neuro- und psychoprotektive Effekte haben - vor allem das Estradiol-17- $\beta$, das natürliche Östrogen, das die stärkste Aktivität im Gehirn zeigt. So gibt es Hinweise darauf, dass Östrogene nicht nur den zerebralen Blutfluss und den Glukose-Metabolismus des Gehirns verbessern, sondern auch das neuronale Wachstum und die Myelinisierung, dass sie die Synapsendichte und -plastizität im Gehirn erhöhen, die neuronale Konnektivität verbessern, anti-oxidativ wirken und den neuronalen Zelltod inhibieren. Außerdem modulieren sie die verschiedensten Neurotransmittersysteme im Gehirn, die für unser psychisches Befinden relevant sind, etwa das serotonerge, das dopaminerge, das glutamerge, das noradrenerge und das cholinerge (Übersicht bei [1-3]).

Klinisch gibt es inzwischen Hinweise auf die verschiedensten positiven Effekte der Östrogene, insbesondere des Estradiol-17- $\beta$, auf das psychische Befinden. Vermutet wird u.a. eine Verbesserung affektiver Symptome, eine antipsychotische Wirkung, die Reduktion aggressiven und suizidalen Verhaltens, eine stressprotektive Wirkung 
sowie eine Verbesserung kognitiver Funktionen (Übersichten bei [1-4]). Fink et al. bezeichneten die Östrogene wegen dieser zahlreichen positiven Effekte als „Psychoschutz der Natur" [5].

\section{Depression und Menopause}

Einer der interessantesten und stabilsten epidemiologischen Befunde zur Depression ist derjenige des Geschlechtsunterschiedes: Die Prävalenz depressiver Störungen ist bei Frauen zwei bis drei Mal so hoch wie bei Männern. Dieser Geschlechtsunterschied beginnt offensichtlich nach der Pubertät.

Für diesen Häufigkeitsunterschied gibt es sicherlich vielfältige psychosoziale Erklärungen [6]. Immer wieder wurde aber auch beobachtet, dass milde depressive Symptome, emotionale Labilität und Irritabilität einen Zusammenhang mit der Fluktuation des Östrogenspiegels zeigen (Übersicht bei [2]). Dies betrifft sowohl das Prämenstruum und die Postpartalzeit, v. a. aber auch die Perimenopause.

Den Gynäkologen bekannt sind die milden depressiven Verstimmungen und die erhöhte emotionale Labilität und Irritabilität einiger Frauen in der Perimenopause, also in der Zeit abfallender Hormonspiegel [2, 3]. Neuere Studien zeigen, dass auch die Inzidenz und Prävalenz schwerer, krankheitswertiger Depressionen in der Perimenopause ansteigen [7-10], und zwar in Korrelation mit den hormonellen Schwankungen, was sehr stark für die biologische Mitverursachung dieses Anstiegs spricht.

Andere Risikofaktoren sind u.a. Hitzewallungen und schlechter Schlaf, was aber ebenfalls durch die hormonellen Änderungen bedingt sein kann, sowie stressvolle oder negative Lebensereignisse. Tabelle 2 gibt eine Übersicht. In der Postmenopause scheint die Prävalenz der Depression bei Frauen stabil zu bleiben oder sogar wieder abzusinken (Übersichten bei [2, 3]).

- Tabelle 2 etwa hier einfügen - 
Interventionsstudien mit Östrogenen bei perimenopausaler Depression zeigen eine gute therapeutische Wirksamkeit, insbesondere bei operativ bedingter Menopause (Übersichten bei [3, 4]), aber auch bei physiologisch auftretender Menopause. So haben Zweifel und O'Brien 1997 [11] 26 Studien einer Metaanalyse unterzogen und konnten zeigen, dass Östrogene zumindest bei milden depressiven Symptomen hilfreich sind und zwar vor allem in der Perimenopause, weniger in der Postmenopause. Neuere Studien zeigen auch eine Effektivität von Östrogengaben bei schwereren perimenopausalen Depressionen, die die DSM-Kriterien erfüllen (Übersicht bei [2, 3, 12]). Kornstein zeigte auch, dass postmenopausale Frauen mit Hormonersatztherapie weniger unter melancholischer Symptomatik leiden [13].

\section{Schizophrene Psychosen und Menopause}

Schon zu Beginn des 20. Jahrhunderts erkannten Psychiater die möglichen Zusammenhänge zwischen Psychosen und Östrogenen (Übersichten bei [14, 15]). Sie beschrieben einerseits Anzeichen eines chronischen "Östrogenmangels" bei Frauen mit Schizophrenie, worauf hier nicht näher eingegangen werden soll. Vor allem aber beschrieben diese frühen Kliniker auch schon den Zusammenhang zwischen hormonellen Schwankungen und psychotischer Symptomatik. So berichtete schon Manfred Bleuler über die Häufung der Spätschizophrenien bei Frauen, also der schizophrenen Ersterkrankungen nach dem 40. Lebensjahr und brachte diese in Zusammenhang mit den hormonellen Schwankungen in dieser Zeit. Die Forschung der letzten Dekaden hat nun weitere Hinweise auf einen möglichen Zusammenhang zwischen Östrogenen und Schizophrenie gebracht.

Wie wir in der ABC-Studie (Age, Begin and Course of Schizophrenia) zeigen konnten [16, 17], haben Frauen mit schizophrenen Psychosen einen deutlich späteren Erkrankungsbeginn als Männer, haben jedoch einen zweiten Erkrankungsgipfel nach dem 45. Lebensjahr. Dieser Befund könnte dadurch erklärt werden, dass die Östrogene bis zum 45. Lebensjahr einen gewissen Schutz vor dem Ausbruch der Erkrankung geben, einen Schutz, der dann aber nach dem 45. Lebensjahr durch das physiologische Sistieren der Estradiolproduktion nachlässt, so dass es danach zu einem „Nachholeffekt“ bei vulnerablen Frauen kommt. 
Frauen in der Postmenopause haben wahrscheinlich dadurch bedingt nicht nur eine etwa doppelt so hohe Inzidenz von schizophrenen Psychosen als Männer derselben Altersgruppe, sondern auch eine deutlich schwerere Symptomatik und einem schlechteren Verlauf [18]. Auch der Verlauf chronischer, vorbestehender Psychosen scheint sich in der Postmenopause zu verschlechtern [19].

Erste Östrogeninterventionsstudien bei Frauen mit Schizophrenie und anderen Psychosen zeigten positive Effekte (Übersicht bei [1, 3, 15]). Nachdem schon Manfred Bleuler erste unsystematische Therapieversuche mit einem Kombinationspräparat aus ovariellen und hypophysären Hormonen unternommen hatte, berichtete auch Mall aus einem deutschen Landeskrankenhaus, dass eine Subgruppe von "hypofollikulären“ schizophreniekranken Frauen „relativ leicht“ durch eine Substitutionstherapie mit Östrogenen (Primodian oder Depot-Progynon) vollständig „geheilt werden kann“.

Inzwischen haben verschiedene Studien bei akut psychotischen jüngeren Frauen zeigen können, dass sich durch eine adjuvante Östrogengabe zusätzlich zu Standard-Neuroleptika eine raschere und bessere Symptombesserung erzielen lässt (Übersicht bei [1, 3, 15, 20]. Einen ähnlichen Effekt berichteten Lindamer et al. über eine postmenopausale Frau. Diese Autorengruppe konnte auch zeigen, dass postmenopausale schizophreniekranke Frauen, die aus medizinischer Indikation eine Östrogensubstitution erhielten, weniger schizophrene Minussymptomatik aufwiesen und eine signifikant geringere Neuroleptikadosis benötigten [21].

\section{Östrogentherapie - eine Option für psychisch kranke Frauen in der Perimenopause?}

Ausgelöst durch die One Million Women Study [22] und dann die Women's Health Initiative (WHI)-Studie [23] ist in den letzten Jahren eine Kontroverse um die postmenopausale Hormon(ersatz)therapie, $H(R) T$, entstanden. Allerdings wurden die Interpretation und v.a. die Verallgemeinerung der Ergebnisse der WHI-Studie z.T. scharf kritisiert [3, 24]. So wurde in dieser Studie nur die prophylaktische, nicht die therapeutische Anwendung von Östrogenen untersucht. Das mittlere Alter der Frauen war bei Behandlungsbeginn mit 63 Jahren extrem hoch. Entsprechend wiesen diese Frauen zahlreiche kardio-vaskuläre und andere Risikofaktoren auf. 
Zahlreiche Konsensusgruppen haben inzwischen trotzdem neue Richtlinien verabschiedet (Übersichten z.B. bei [24, 25]). Fazit all dieser Richtlinien ist immer wieder, dass unter Beachtung der Kontraindikationen und der individuellen NutzenRisiko-Analyse die Indikation für jede Frau äußerst sorgfältig zu stellen ist (vgl. Tab. 3). Die meisten Fachgesellschaften empfehlen keine langfristige Östrogengabe mehr und betonen das „window of opportunity“: Der Hormonersatz soll unmittelbar in oder möglichst früh nach Eintritt der Menopause starten. Dann überwiegen die positiven die potentiell negativen Wirkungen.

- Tabelle 3 etwa hier einfügen -

Wichtig scheint aber vor allem auch, bei der Indikationsstellung und Risikoabwägung zu unterscheiden zwischen vorübergehenden Hormongaben aus therapeutischen Gründen (einer Hormonersatztherapie im engeren Sinne etwa bei perimenopausalen Beschwerden) und langfristigen Hormongaben aus prophylaktischen Gründen (bei symptomfreien Frauen, etwa zur Vorbeugung von Osteoporose).

In der Psychiatrie geht es nicht um einen prophylaktischen Einsatz, bei dem die Sicherheitsanforderungen ganz besonders hoch sein müssen, sondern um den therapeutischen Einsatz bei bestehenden Beschwerden. Hier müssen die Östrogene also lediglich dem Vergleich $\mathrm{zu}$ anderen Behandlungsmethoden, etwa Psychopharmaka, standhalten.

\section{Therapie der Depression in der Menopause}

Insbesondere bei depressiven Erkrankungen in der Perimenopause könnte eine Estradiolsubstitution sinnvoll sein. Allerdings gibt es für diese Indikation noch keine Zulassung. Wenn es zusätzliche Indikationen für eine Östrogensubstitution gibt (z. B. Hitzewallungen oder erhöhtes Osteoporose-Risiko), wäre ein solcher Therapieversuch aber sicherlich gerechtfertigt. Voraussetzung ist selbstverständlich, dass keine Risikofaktoren oder Kontraindikationen für die Östrogensubstitution bestehen, und dass eine gut informierte Frau die Östrogensubstitution wünscht. Bei leichteren Depressionen in der Perimenopause könnte Estradiol-17- $\beta$ bei gegebenen Zusatzindikationen noch vor Antidepressiva eingesetzt werden, wie dies 
Gynäkologen ja auch z. T. schon lange praktizieren. Antidepressiva würden in diesen Fällen nur dann hinzugegeben, wenn eine Estradiolsubstitution alleine nicht ausreichend ist (Tab. 4).

Bei schwererer Depression sind Antidepressiva auch in der Perimenopause Mittel erster Wahl, aber auch hier könnte eine zusätzliche, „adjuvante“ Verordnung von Estradiol-17- $\beta$ hilfreich sein (Tab. 4). In diesem Zusammenhang interessant sind Hinweise darauf, dass Frauen mit Östrogenersatztherapie besser auf Antidepressiva vom SSRI-Typ ansprechen, wahrscheinlich da beide Substanzgruppen die serotonerge Aktivität im Gehirn erhöhen [26].

- Tabelle 4 etwa hier einfügen -

Andererseits scheinen bestimmte Antidepressiva wie Fluoxetin, Paroxetin, Citalopram oder Venlafaxin gegen Hitzewallungen wirksam zu sein, auch wenn keine Depression vorliegt. Ein Einsatz dieser Antidepressiva vom Typ der SSRI (SerotoninReuptake-Inhibitors) bzw. SNRI (Serotonin-Noradrenalin-Reuptake-Inhibitors) ist deshalb zur Therapie von Hitzewallungen zu erwägen, wenn eine Estradiolsubstitution nicht indiziert ist (Übersicht bei [2, 3]).

Auch der Psychotherapie kommt in dieser Lebensphase eine wichtige Bedeutung zu, da Frauen in dieser Zeit häufig sehr vielen psychosozialen Belastungen ausgesetzt sind. So werden sie durch die Menopause nicht nur mit dem Verlust ihrer Fruchtbarkeit konfrontiert, sondern haben sich auch mit dem Übergang in eine neue Lebensphase auseinanderzusetzen. Häufig bestehen große Ängste in Bezug auf den eigenen Körper, das Altern, die Attraktivität, die Sexualität etc.. Gleichzeitig erleben Frauen in dieser Zeit oft zahlreiche äußere Belastungen und Verluste, etwa durch die Pflege der Eltern oder deren Tod, den Wegzug der erwachsen gewordenen Kinder, den Verlust des Partners oder eine Verschlechterung der eigenen körperlichen Gesundheit.

Die Abklärung und Behandlung einer Depression bei Frauen nach dem 40./45. Lebensjahr sollte also unbedingt eine ganzheitliche sein, die sowohl die hormonellen und anderen körperlichen Aspekte als auch die psychiatrisch-psychotherapeutischen 
Möglichkeiten umfasst. Wichtig ist dabei, dass vor einer Therapie immer eine gute Abklärung stehen sollte, bei der andere Ursachen, wie etwa SchilddrüsenFunktionsstörungen etc. ausgeschlossen werden. Eine optimale Abklärung und Behandlung ist oft nur in Zusammenarbeit zwischen Frauenarzt und Psychiater möglich.

\section{Therapie schizophrener Psychosen in der Perimenopause}

Was schizophreniekranke Frauen betrifft, so wäre auch bei innen v.a. in der Perimenopause eine Östrogensubstitution zu erwägen. Insbesondere wenn bei einer Frau auch andere Indikationen für eine Östrogensubstitution bestehen, könnte die Schizophrenie eine zusätzliche Indikation darstellen, zum einen wegen der vermuteten antipsychotischen Effekte, zum anderen aber auch wegen der möglichen positiven Effekte auf die Kognition, die Affekte, die Stressvulnerabilität, die Aggressivität und Suizidalität. Durch die Hormonsubstitution könnten unter Umständen Neuroleptika und deren Nebenwirkungen reduziert werden.

Für beide Diagnosegruppen von Vorteil wäre, dass eine Östrogensubstitution die klimakterischen Beschwerden wie Hitzewallungen, Schlafstörungen, Irritabilität etc. bessern kann, die bei schizophreniekranken Frauen durchaus als rückfallprovozierende Stressoren in Frage kommen und über den sog. Dominoeffekt auch eine Depression auslösen oder verstärken können.

\section{Vorzeitige Menopause bei psychisch kranken Frauen}

Nicht zu vernachlässigen ist in diesem Zusammenhang schließlich, dass eine Neuroleptika- oder auch Antidepressiva-Gabe zu einer vorzeitigen Menopause führen kann [27, 28]. Insbesondere viele Prolaktin-erhöhende Neuroleptika können zu einer massiven gonadalen Suppression führen. Das heißt, dass die Menopause bei so behandelten psychisch kranken Frauen oft deutlich früher eintritt als bei psychisch gesunden Frauen. Bei einem solchen Verdacht sollten Psychiater und Gynäkologen eng zusammenarbeiten. 


\section{Kooperationsbedarf Psychiatrie - Gynäkologie}

Ein sehr enger Kooperationsbedarf zwischen Gynäkologie und Psychiatrie besteht auch bezüglich der Indikationsstellung für eine Östrogen-Therapie. Risikofaktoren und Kontraindikationen des Östrogenersatzes sind durch die GynäkologInnen sehr gut abzuklären. Gemeinsam ist eine sehr sorgfältige Nutzen-Risiko-Abwägung durchzuführen und zwar für jeden Individualfall. Eine Depression oder eine Psychose können in dieser Abwägung unter Umständen aber als zusätzliches Argument für eine Östrogenersatztherapie gewertet werden.

\section{Forschungsbedarf}

Viele Fragen im Bereich der Anwendung von Östrogenen in der Psychiatrie bleiben noch offen. So bedarf es z. B. dringend der Forschung bezüglich der besten Art des Hormonersatzes für psychisch Kranke. Vor allem für Estradiol-17- $\beta$ wurden neuropsychoprotektive Effekte gezeigt. Estradiol-17- $\beta$ wäre also bei psychischen Störungen indiziert. Dabei sollte wegen des günstigeren Nebenwirkungsprofils eventuell die transdermale Applikation (z. B. Pflaster oder Gel) bevorzugt werden.

Bei Frauen, die noch einen Uterus haben, müssen die Östrogene zur Karzinomprophylaxe mit einem Gestagen kombiniert werden. Gestagene können aber die positiven Effekte der Östrogene bezüglich des psychischen Befindens z.T. antagonisieren [29]. Hier ist eine sorgfältige Wahl des Gestagens zu treffen und z.B. Medroxyprogesteron oder Dydrogesteron vorzuziehen [30].

Auch wäre dringender Forschungsbedarf bezüglich der Kontraindikationen bei therapeutischem Gebrauch und der relativen Risiken im Vergleich zu Psychopharmaka gegeben.

Weitere Studien müssten sich auf die geringste wirksame Dosis sowie auf die Kombination einer Östrogen(ersatz)-Therapie mit Psychopharmaka beziehen, um mögliche Augmentierungsstrategien und Interaktionen zu untersuchen.

Schließlich wären selektive Östrogenrezeptormodulatoren, SERMS, besser im Hinblick auf psychoaktive Wirkungen zu prüfen . 


\section{Schlussfolgerungen}

Frauen in der Perimenopause haben sehr spezifische diagnostische und therapeutische Bedürfnisse. Diese sollten sowohl die verschiedenen psychosozialen, als auch die verschiedenen biologischen und hormonellen Veränderungen berücksichtigen. Nicht alle, aber viele Frauen entwickeln in dieser Zeit psychische Störungen, vorbestehende psychische Störungen exazerbieren häufig. Die Therapie in dieser Zeit sollte neben der üblichen psychiatrisch-psychotherapeutischen Behandlung immer auch die hormonelle Situation berücksichtigen. Da Östrogene wichtige psychoprotektive Eigenschaften haben, kann ihr Verlust bei vulnerablen Frauen wahrscheinlich psychische Erkrankungen triggern oder aggravieren. Eine Östrogensubstitution kann in solchen Fällen nach sorgfältiger Nutzen-RisikoAbwägung hilfreich sein.

Mehr Forschung über Indikation und Kontraindikation der Hormonersatztherapie bei psychisch kranken Frauen wäre dringend erforderlich.

$\mathrm{Zu}$ hoffen wäre, dass die Forschung der künftigen Jahre die empirische Evidenz in diesem Bereich weiter verbessert, so dass ideologische Kontroversen mehr und mehr durch klare, empirisch fundierte Leitlinien ersetzt werden können. Dabei gibt es berechtigte Hoffnung, dass Östrogene als neuroprotektive und psychoprotektive adjuvante Therapiestrategien die traditionelle Psychopharmakotherapie bei psychischen Erkrankungen in Zukunft ergänzen werden.

In der Klinik könnte schon jetzt durch eine engere Kooperation zwischen Gynäkologie und Psychiatrie sehr viel für unsere Patientinnen erreicht werden. 


\section{Literatur}

1 Bergemann N, Riecher-Rössler A: Estrogen effects in psychiatric disorders. Wien, New York, Springer, 2005.

Riecher-Rössler A, Kuhl H, Bitzer J. Psychische Störungen in Zeiten hormoneller Umstellung bei Frauen - Eine selektive Übersicht. Neuropsychiatrie, 20: 155-165, 2006.

3 Riecher-Rössler A, de Geyter C. The forthcoming role of treatment with oestrogens in mental health. Swiss Med Wkly, 137: 565-572, 2007.

$4 \quad$ Kahn L, Halbreich U. Estrogen's effect on depression; in Bergemann N, Riecher-Rössler A (eds): Estrogen effects in psychiatric disorders. Wien, New York, Springer, pp 145-173, 2005. Fink G, Sumner BEH, Rosie R, et al. Estrogen control of central neurotransmission: effect on mood, mental state, and memory. Cell Mol Neurobiol, 16: 325-344, 1996.

Bitzer J, Riecher-Rössler A. Was sind Frauenleiden? Epidemiologische Aspekte; in RiecherRössler A, Bitzer J (Hrsg.): Frauengesundheit. Ein Leitfaden für die ärztliche und psychotherapeutische Praxis. München, Elsevier Urban \& Fischer, pp 13-29, 2005. Cohen LS, Soares CN, Vitonis AF, et al. Risk for new onset of depression during the menopausal transition: the Harvard study of moods and cycles. Arch Gen Psychiatry, 63: 385390, 2006.

Freeman EW, Sammel MD, Lin H, et al. Associations of hormones and menopausal status with depressed mood in women with no history of depression. Arch Gen Psychiatry, 63: 375382, 2006.

9 Bromberger JT, Kravitz HM, Chang YF, et al. Major depression during and after the menopausal transition: Study of Women's Health Across the Nation (SWAN). Psychol Med: 110, 2011.

Bromberger JT, Schott LL, Kravitz HM, et al. Longitudinal change in reproductive hormones and depressive symptoms across the menopausal transition: results from the Study of Women's Health Across the Nation (SWAN). Arch Gen Psychiatry, 67: 598-607, 2010. Zweifel JE, O'Brien WH. A meta-analysis of the effect of hormone replacement therapy upon depressed mood. Psychoneuroendocrinology, 22: 189-212, 1997.

Birkhäuser M. Depression und Östrogene. Besteht eine kausale Beziehung? Gynäkol Endokrinol, 8: 82-88, 2010.

Kornstein SG, Young EA, Harvey AT, et al. The influence of menopause status and postmenopausal use of hormone therapy on presentation of major depression in women. Menopause, 17: 828-839, 2010.

14 Riecher-Rössler A. Psychotic disorders and menopause: the untold story; in Soares C, Warren $M$ (eds): The Menopausal Transition Interface between Gynecology and Psychiatry Key Issues in Mental Health. Basel, Karger, vol 175, pp 115-126, 2009.

Riecher-Rössler A. Estrogens and schizophrenia; in Bergemann N, Riecher-Rössler A (eds): Estrogen Effects in Psychiatric Disorders. Wien, Springer, pp 31-52, 2005. the results. Soc Psychiatry Psychiatr Epidemiol, 33: 380-386, 1998. Riecher A, Maurer K, Löffler W, et al. Gender differences in age at onset and course of schizophrenic disorders; in Häfner H, Gattaz WF (eds): Search for the causes of schizophrenia. Berlin, Heidelberg, Springer, vol 2, pp 14-33, 1991. Riecher-Rössler A, Löffler W, Munk-Jorgensen P. What do we really know about late-onset schizophrenia? Eur Arch Psychiatry Clin Neurosci, 247: 195-208, 1997.

19 Riecher-Rössler A, Rössler W. The course of schizophrenic psychoses: what do we really know? A selective review from an epidemiological perspective. Eur Arch Psychiatry Clin Neurosci, 248: 189-202, 1998.

20 Riecher-Rössler A, Kulkarni J. Estrogens and gonadal function in schizophrenia and related psychoses in Neill JC, Kulkarni J (eds): Biological Basis of Sex Differences in Psychopharmacology. Berlin, Heidelberg Springer, vol 8, pp 155-171, 2011. Lindamer LA, Buse DC, Lohr JB, et al. Hormone replacement therapy in postmenopausal women with schizophrenia: positive effect on negative symptoms? Biol Psychiatry, 49: 47-51, 2001.

Million Women Study C. Breast cancer and hormone-replacement therapy in the Million Women Study. Lancet: 419-427, 2003.

Rossouw JE, Prentice RL, Manson JE, et al. Postmenopausal hormone therapy and risk of cardiovascular disease by age and years since menopause. JAMA, 297: 1465-1477, 2007. Birkhäuser MH, Panay N, Archer DF, et al. Updated practical recommendations for hormone replacement therapy in the peri- and postmenopause. Climacteric, 11: 108-123, 2008. 
25 Santen RJ, Allred DC, Ardoin SP, et al. Postmenopausal hormone therapy: an Endocrine Society scientific statement. J Clin Endocrinol Metab, 95: s1-s66, 2010.

26 Morgan ML, Cook IA, Rapkin AJ, et al. Estrogen augmentation of antidepressants in perimenopausal depression: a pilot study. J Clin Psychiatry, 66: 774-780, 2005.

27 Harlow BL, Wise LA, Otto MW, et al. Depression and its influence on reproductive endocrine and menstrual cycle markers associated with perimenopause: the Harvard Study of Moods and Cycles. Arch Gen Psychiatry, 60: 29-36, 2003.

28 Riecher-Rössler A, Schmid C, Bleuer S, et al. Antipsychotika und Hyperprolaktinämie: Pathophysiologie, klinische Bedeutung, Abklärung und Therapie. Neuropsychiatr, 23: 71-83, 2009.

29 Kuhl H. Psychoendokrinologische Einflussfaktoren; in Riecher-Rössler A, Bitzer J (Hrsg.): Frauengesundheit. Ein Leitfaden für die ärztliche und psychotherapeutische Praxis. München, Elsevier Urban \& Fischer, pp 51-62, 2005.

30 Cagnacci A, Arangino S, Baldassari F, et al. A comparison of the central effects of different progestins used in hormone replacement therapy. Maturitas, 48: 456-462, 2004.

31 Riecher-Rössler A. Psychoprotektive Effekte von Östrogenen. J Neurol Neurochir Psychiatr, 12: 152-156, 2011. 
Tabelle 1: Definitionen Menopause, Perimenopause, Postmenopause*

$\begin{array}{ll}\text { Menopause } & \begin{array}{l}\text { Dauerhaftes Sistieren der Menstruation infolge des Ausbleibens der } \\ \text { ovariell- follikulären Aktivität } \\ \text { (mindestens } 12 \text { Monate Amenorrhoe) }\end{array}\end{array}$

Perimenopause Zeitraum unmittelbar vor der Menopause

- wenn endokrinologische, biologische und klinische Kennzeichen der bevorstehenden Menopause beginnen

- bis zum Ende des ersten Jahres nach der Menopause

Postmenopause Nach der Menopause

* gemäss World Health Organisation 1996 
Tabelle 2: Risikofaktoren der Depression in der Perimenopause

- Frühere Depression

- Zugehörigkeit zu einer für Östrogen-Abnahme sensiblen Untergruppe

- frühere postpartale Depression, prämenstruelle Dysphorie (PMDD)

- Chirurgisch bedingte Menopause

- Späte oder verlängerte menopausale Transition

- Hitzewallungen, Schlafstörungen, vasomotorische Symptome

- Psychosoziale Faktoren:

○ Änderungen in der Familienstruktur

- Verluste

○ Rollenwandel

- stressreiche oder negative Lebensereignisse

- niedriger sozialer Support

Literatur bei der Verfasserin 
Tabelle 3: Einige wichtige Effekte der Östrogensubstitution

\begin{tabular}{|c|c|}
\hline \multicolumn{2}{|c|}{ Einige wichtige Effekte der Östrogensubstitution } \\
\hline Positive & Negative \\
\hline $\begin{array}{l}\text { - Perimenopausale Beschwerden vermindert } \\
\text { körperlich: weniger Hitzewallungen, } \\
\text { genitale Beschwerden, Kollagenalterung } \\
\text { (Haut, Gelenke, Bandscheiben) } \\
\text { psychisch: weniger Depression, Irritabilität, } \\
\text { emotionale Labilität }\end{array}$ & $\begin{array}{l}\text { - Endometriumkarzinomrisiko erhöht bei alleiniger } \\
\text { Östrogengabe } \\
\text { (Wenn Uterus noch vorhanden, nur in } \\
\text { Kombination mit Gestagenen verordnen!) }\end{array}$ \\
\hline - Osteoporoserisiko gesenkt & $\begin{array}{l}\text { Risiko für Mammakarzinom erhöht, } \\
\text { wahrscheinlich durch Gestagene? } \\
\text { (Nicht bei Risikopatientinnen und nicht länger als } \\
7 \text { Jahre verordnen oder dann in reduzierter } \\
\text { Dosis!) }\end{array}$ \\
\hline $\begin{array}{l}\text { - Verzögerung kognitiver Störungen/Morbus } \\
\text { Alzheimer? }\end{array}$ & $\begin{array}{ll}\text { - } & \text { Risiko für Thrombose und Schlaganfall erhöht } \\
\text { (Nicht bei Risikopatientinnen verordnen!) }\end{array}$ \\
\hline $\begin{array}{l}\text { - Kardiovaskuläre Protektion? } \\
\text { (wenn Beginn unmittelbar nach Menopause) }\end{array}$ & $\begin{array}{l}\text { - Kardiovaskuläre Risiken (Arteriosklerose, KHK) } \\
\text { erhöht? } \\
\text { (Nur innerhalb der ersten } 10 \text { Jahre nach der } \\
\text { Menopause beginnen und nicht bei schon } \\
\text { bestehenden kardiovaskulären Erkrankungen!) }\end{array}$ \\
\hline
\end{tabular}

Übersichten bei [2, 3, 14, 20, 24, 25, 31] 
Tabelle 4: Therapie der Depression für Frauen in der Perimenopause

\begin{tabular}{|c|c|}
\hline $\begin{array}{l}\text { Östrogen- } \\
\text { Ersatztherapie }\end{array}$ & $\begin{array}{l}\text { - } \quad \text { in der Perimenopause (nicht in der Postmenopause?) } \\
\text { - } \quad \text { insbesondere bei erstmaligem Auftreten in der Perimenopause? } \\
\text { - } \quad \text { bepression in der Vorgeschichte) } \\
\text { - } \quad \text { falls keine Risikofaktoren/Kontraindikationen vorliegen } \\
\text { - } \quad \text { bei gut informierten Frauen mit Wunsch nach Östrogenen } \\
\text { - } \quad \text { bei zusätzlichen Indikationen für Östrogenersatz } \\
\text { (da bisher keine Zulassung für Depression!) }\end{array}$ \\
\hline $\begin{array}{l}\text { Antidepressiva } \\
\text { (z.B. SSRI) }\end{array}$ & $\begin{array}{l}\text { - bei mittelschwerer bis schwerer Depression oder } \\
\text { - wenn } E_{2} \text {-Ersatz kontraindiziert oder ungenügend ist }\end{array}$ \\
\hline & dere mit SSRI scheint vielversprechend \\
\hline
\end{tabular}

Literatur siehe Text und bei der Verfasserin 